\begin{tabular}{c} 
International Journal of Engineering \& Technology, 7 (4.36) (2018) 506-510 \\
International Journal of Engineering \& Technology \\
SPC \\
Website: www.sciencepubco.com/index.php/IJET \\
Research paper \\
\hline
\end{tabular}

\title{
Performance of Bluetooth in Homogeneous Environment
}

\author{
A. S. Shirsat1 ${ }^{1}$, S. A. Shirsat ${ }^{2}$, D. M. Yadav ${ }^{3}$ \\ ${ }^{1}$ Research scholar, AISSMSIOIT, SSPU, Pune, India \\ ${ }^{2}$ Assistant Professor Sinhgad College of Engineering Pune, India \\ ${ }^{3}$ Principal, Universal College of Engg., Pune, India \\ *Corresponding author E-mail:asshirsat@gmail.com
}

\begin{abstract}
Various wireless technologies use unlicensed ISM band in a frequency range 2.4GHz. Due to collocated, services causes mutual interference to one other; it degrades performance regarding Bit Error Rate (BER) and consequently throughput. The Bluetooth occupies less bandwidth and low power, gets more hampered as compare to other high bandwidth services is referred to as the heterogeneous environment. In this paper, an attempt is made to calculate the performance of basic rate Bluetooth in the presence of other Bluetooth devices in the close vicinity. The MATLAB based simulation results showed the degradation of performance of Bluetooth and enriched by selecting different hopping frequency in synchronisation with interfering Bluetooth devices.
\end{abstract}

Keywords: Adaptive Frequency Hopping, Bit Error Rate, Homogeneous, Throughput, WLAN.

\section{Introduction}

Bluetooth is a standard wireless technology available in various devices, due to low cost, low power short range wireless communication $[1,2]$. It can easily connect to various devices such as computers, cameras, notebook, headsets, mobile phones, wireless printers and other Bluetooth enabled devices wirelessly. Bluetooth operates in license-free Industrial Scientific and Medical $2.4 \mathrm{GHz}$ band, where many radio technologies present such as WLAN, ZigBee, cordless phones [3, 4]. Bluetooth designed to operate in a noisy frequency environment, with fast acknowledgement and frequency hopping technique to mitigate the interference in the band. Bluetooth radio hops faster than other devices $[5,6]$.

Various work reported for Bluetooth performance regarding BER, PER and throughput in the presence of other radio access technologies such as ZigBee and WLAN. The performance of Bluetooth degrades rapidly by WLAN, which is also a function of distance. However, it is required to study and check the performance of basic rate Bluetooth in the presence of other Bluetooth devices in the close vicinity. In this work main focus is to the performance of Bluetooth regarding BER and throughput inhomogeneous network. From the result obtained it is clear that the BER and throughput of Bluetooth reduction with an increase in interfering Bluetooth. There is a tradeoff between the distance of interfering Bluetooth with BER and throughput. The performance can be enhanced by applying a different hopping sequence to Bluetooth.

This paper is organised as follows. Section II provides a review of the literature. Overview of Bluetooth is given in section III. Section IV introduces homogeneous interference model of the proposed system. Results obtained are presented in Section V. Finally we conclude in section VI.

\section{Related Work}

Bluetooth, ZigBee and WLAN operate in the same ISM licensefree band, so the coexistence between these services. As the devices differ in physical design and protocol aspect, this leads signal of one acts as interference to another device. Since the Bluetooth and WLAN protocol based on different mechanism, a signal of these is not mutually understandable to one other. It affects the performance regarding BER and throughput. Wi-Fi checks the channel availability and then transmits the signal, whereas Bluetooth continues its transmission irrespective of channel availability. Though the WLAN has large bandwidth than Bluetooth, WLAN suffers more than Bluetooth regarding throughput [7-11].

Various work reported in co-existence problem of Bluetooth and WLAN. Some work related to the network level and few in device level. Most of the work addresses interference management on network level co-existence. In [12] this different adaptive frequency hopping techniques used to mitigate the co-existence issues. An interference source oriented adaptive frequency hopping [ISOAFH] approach given by Check et al. Hsu et al. proposed dynamic packet fragmentation to enhance throughput of WLAN in the presence of Bluetooth devices in the closed vicinity [13]. Overlap Avoidance (OLA) method, where packet length adjusted in Bluetooth and Wi-Fi introduced by Chiasserini and Rao [14]. In some work reported in sharing the antenna for this protocol by time division scheduling technique [15]. M. A. M. Mohamed et al. employed convolution codes in the payload to enhance the performance for both AWGN and Rayleigh channel. Convolution code can better for large length payload [16]. Seung Hwan Lee and Yong Hwan Lee calculated performance of Bluetooth using Adaptive Frequency Hopping AFH scheme by dividing Bluetooth channel concerning WLAN as a good channel [17].

The author proposed new scheme to improve throughput performance of Bluetooth over good channel condition the Channel Quality Driven Data Rate (CQDDR) is determined and then pack- 
ets used for transmission, but it fails for larger packets [18]. M. A. M. El Bendary and M. A. R. El Tokhy proposed a new technique to reduce retransmission time with error control scheme for EDR Bluetooth packets, but at higher values of $\mathrm{Eb} / \mathrm{No}$ effect is not up to the mark [19]. Li Jing et al. proposed adaptive packet selection strategies by estimating the SNR of the Bluetooth channel. The method is suitable for different modulation scheme and data packets [20]. The author proposed BlueCode to mitigate the coexistence in the dense environment to enrich throughput of WLAN up to $12 \mathrm{Mbps}$ in Bluetooth by coordination scheme for multiple Bluetooth devices [21].

From the above survey, it is clear that work reported addresses the performance of Bluetooth in the heterogeneous wireless environment $[22,23]$. However, it is required to study and evaluate the performance of Bluetooth in a homogeneous environment.

\section{Overview of Bluetooth}

Bluetooth a wireless technology used for short-range radio links intended to replace cables in connection with various Bluetooth enabled electronic devices. It envisaged that it would allow for the replacement of many propriety cables that connect one device to other with a universal radio link. It allows the wireless connectivity in unlicensed $2.4 \mathrm{GHz}$ ISM band. The key features of Bluetooth are robustness, low complexity, low power and low cost. Designed to operate in noisy environments, Bluetooth uses a fast acknowledgement and frequency-hopping scheme to make the link robust. Bluetooth avoids interference from other signals by hopping to a new frequency after transmitting or receiving packet. Bluetooth radio hops faster and uses shorter wavelengths as compared to other radio systems in the same band. Thus Bluetooth is a wireless LAN technology designed to connect devices of different functions such as telephone, computers, printers, cameras, mobiles and other devices. Bluetooth LAN can connect to the internet, based on IEEE 802.15.1 standard. Table 1 gives the technical specifications of basic rate Bluetooth. Main features consist of the Frequency range, Transmission power, Bluetooth technology, data rate along with Bandwidth utilisation [1].

Table 1 General Specifications of Bluetooth Technology.

\begin{tabular}{|c|c|c|}
\hline Sr. No. & Specification & Feature Supported \\
\hline 1. & RF Frequency & $2.4 \mathrm{GHz}$ \\
\hline 2. & Transmit power & $1 \mathrm{~mW}$ \\
\hline 3. & Data rate & About $1 \mathrm{Mbps}$ \\
\hline 4. & Distance & 100 meter(max.), 1 meter(min.) \\
\hline 5. & Modulation type & Gaussian FSK (GFSK) \\
\hline 6. & Modulation index & Between 0.28 and 0.35 \\
\hline 7. & Frequency deviation & $+/-140$ to $+/-175 \mathrm{KHz}$ \\
\hline 8. & BT product & 0.5 \\
\hline 9. & Number of RF carriers & 79 (max.), 23(min.) \\
\hline 10. & hopping rate & 1600 hops per second \\
\hline 11. & Access type & FH-TDD-TDMA \\
\hline 12. & Frequency Range & $\begin{array}{l}2402-2483.5 \mathrm{MHz} \text { ( } 79 \mathrm{MHz} \text { band) } \\
\text { (23 MHz in Spain) }\end{array}$ \\
\hline
\end{tabular}

GFSK Modulation scheme is used in Bluetooth. The general impulse response of Gaussian filters in the time domain is given by the Eq. 01 .

$h(t)=\frac{1}{2 \pi} \int_{-\infty}^{\infty} H(\omega) e^{i \omega t} d \omega=e^{-\frac{1}{2}\left(\frac{t}{\tau}\right)^{2}}$

Where $H(\omega)=\sqrt{2 \pi} \tau e^{-\frac{1}{2}(\tau \omega)^{2}}$

$$
\tau \text { - Constant }
$$

Time domain representation of GFSK signal is as follows

$S_{G F S K}(t)=\operatorname{Re}\left[\left(\sqrt{\frac{2 E_{b}}{T_{b}}} e^{j 2 \pi m i \Delta f t}\right) e^{j 2 \pi f_{c} t}\right]$

$S_{G F S K}(t)=\sqrt{\frac{2 E_{b}}{T_{b}}} \cos \left(2 \pi f_{c} t+2 \pi m i \Delta f t\right)$

Where $1 \leq \mathrm{mi} \leq \mathrm{M}$ and $0 \leq \mathrm{t} \leq \mathrm{T}_{\mathrm{b}}$,

mi modulation index and $\mathrm{Tb}$ bit period. Here carrier shifted by mi $\Delta f$.

Let $\quad d(t)=\sum_{n=0}^{n} I_{n} g\left(\tau-n T_{b}\right)$ be the signal, $g(\mathrm{t})$ be the rectangular pulse with amplitude $1 / 2 \mathrm{~Tb}$ and $\mathrm{I}_{\mathrm{n}}$ amplitude obtained by mapping. The complex baseband transmitted signal will be given by the Eq. 04 .

$S_{T R}(t)=\operatorname{Re}\left[A e^{j \varphi_{m}(t)}\right]$

Here A represents the amplitude of the transmitted signal and $\varphi_{m}(t)$ integrated phase given in Eq. 5 .

$\varphi_{m}(t)=\pi m i \sum_{-\infty}^{t}\left[I_{n} g\left(\tau-n T_{b}\right) d \tau\right]+\varphi_{0}$

Where

$I_{n}$ - Mapped to \pm 1 as per binary input data

$\varphi_{0}$ - Initial phase of the carrier signal, $\varphi_{0}=0$ to avoid the loss of generality.

\section{Block Diagram}

Fig.1. shows the proposed block diagram of Bluetooth Transceiver. At the transmitter, the packets get formed with payload along with the header and trailer information. These packets encoded and then modulated. This data is transmitted over the AWGN channel at $1 \mathrm{~mW}$ output power. Hopping sequence generated and synchronised at transmitter and receiver. At receiver, the signal decoded and checked for the bit error performance in the presence of 1,2 and 3 Bluetooth interferers. MATLAB based simulation is carried out for BER performance. Bit error rate is used to calculate data throughput with different Bluetooth interferer.

\section{Methodology}

MATLAB Based simulation of Bluetooth is carried out regarding BER and calculated throughput. Proposed work evolves the study of Baseband protocol to calculate the data rate and the inhomogeneous throughput environment, for Bluetooth receiver with different Bluetooth interferers. A trade-off between Eb/No and BER, Eb/No and throughput are calculated in one, two and three Bluetooth interferer.

The proposed work completed as 
- Simulation of Bluetooth Transreceiver model in MATLAB Simulink to study the transmission parameters.

- Evaluated the BER and throughput of Bluetooth inhomogeneous network.

Calculated BER and throughput concerning $\mathrm{Eb} / \mathrm{No}$, for basic rate Bluetooth with different Bluetooth interferer.

\section{Results and Discussions}

This section presents the BER performance comparison of basic rate Bluetooth and Bluetooth with the homogeneous network. It can be observed form the Fig. 2 that basic rate Bluetooth without interference outperforms the Bluetooth with interference. It shows an $\mathrm{Eb} / \mathrm{No}$ performance gain of $2.13 \mathrm{~dB}, 4.75 \mathrm{~dB}$ and $6.67 \mathrm{~dB}$ compared to Bluetooth with a Bluetooth interference, two Bluetooth interferences and three Bluetooth interferences respectively.

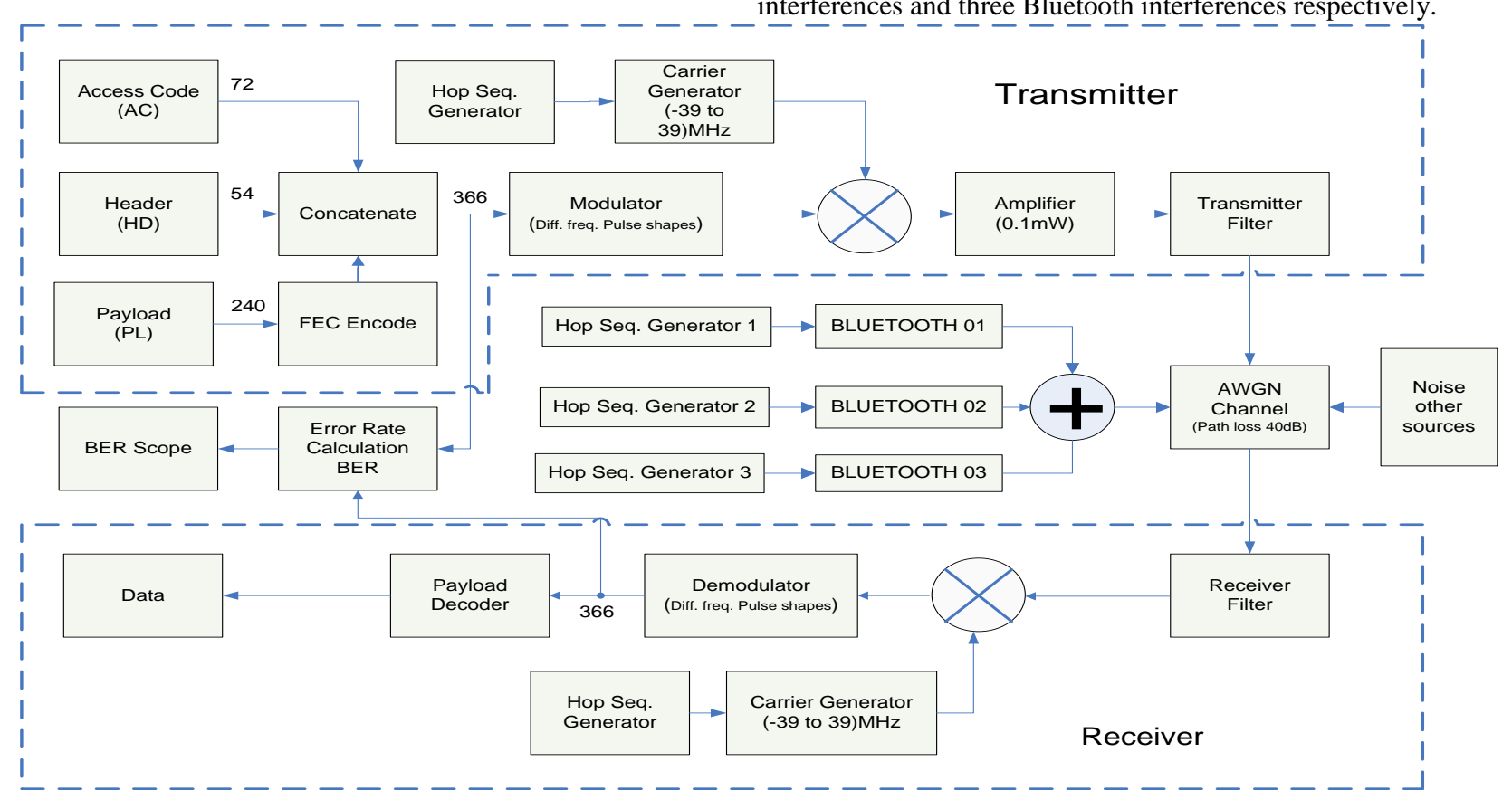

Fig. 1: Block Schematic of Bluetooth Transceiver.

When target BER of $10^{-3}$ is considered without changing the hopping sequence. If hopping sequence of basic rate Bluetooth and interfering Bluetooth is changed, it shows improvement in the BER performance of Bluetooth as depicted in Fig. 3. It shows a significant $\mathrm{Eb} / \mathrm{No}$ gain of $0.1 \mathrm{~dB}$ and $0.5 \mathrm{~dB}$ in the presence of one Bluetooth and 3 Bluetooth interferers respectively.

Fig. 4-6 presents a performance evaluation of Bluetooth regarding throughput for a different number of Bluetooth interferers. Throughput degrades as the number of interferers increases. Fig. 5 shows the effect of changing the hopping sequence on the throughput performance of Bluetooth. It offers substantial enhancement in the throughput as compared to constant hopping sequence as shown in Fig. 4.

Basic rate Bluetooth achieves maximum throughput of $64 \mathrm{Kbps}$ for audio packets at $18 \mathrm{~dB} \mathrm{~Eb} / \mathrm{No}$, whereas it requires $20 \mathrm{~dB}, 22 \mathrm{~dB}$ and excess $24 \mathrm{~dB}$ of $\mathrm{Eb} / \mathrm{No}$ to obtain the same throughput with single interfere, two interferes and three interferes respectively for the same hopping sequence. Basic rate Bluetooth provides a

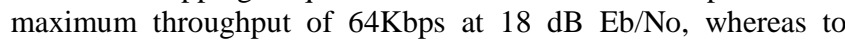
achieve the same throughput with single interfere, two interferes and three interferes it requires nearly same $18 \mathrm{~dB} \mathrm{~Eb} / \mathrm{No}$ under the different hopping sequence scenario. Simulation results reveal that different hopping sequence scheme performs well in the homogeneous environment.

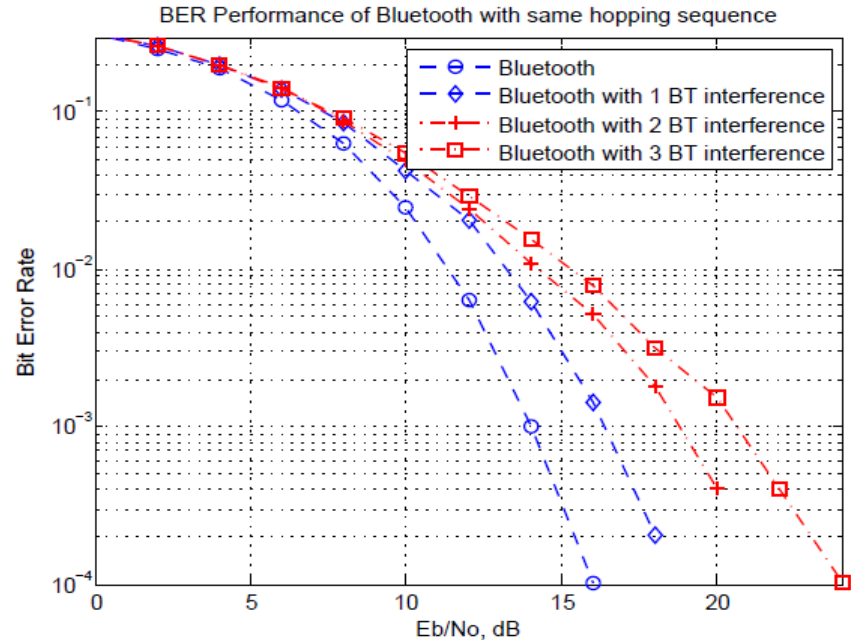

Fig. 2: BER Performance of Basic Bluetooth without Interference. 


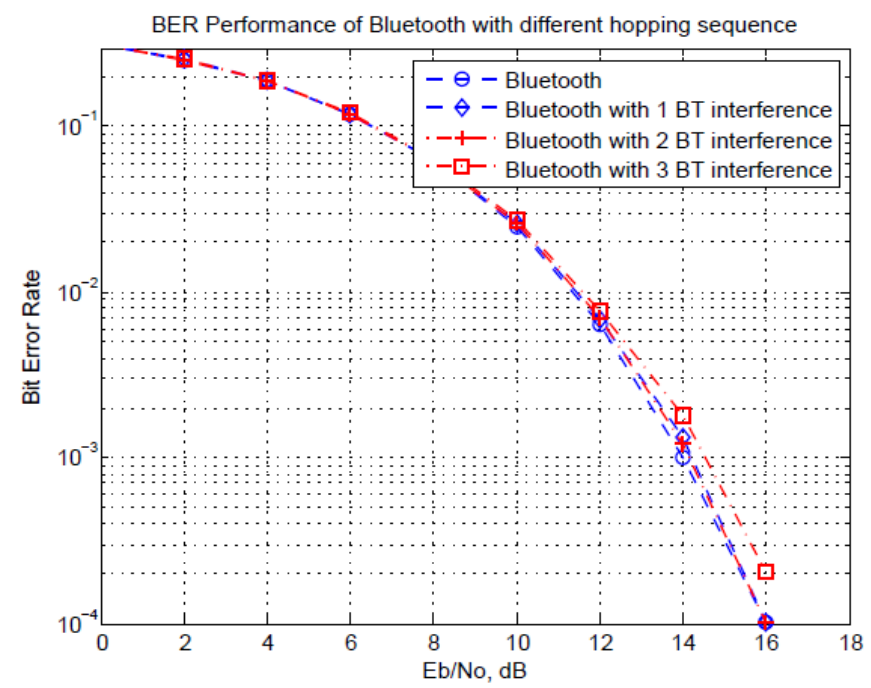

Fig. 3: BER Performance of Basic Bluetooth with Bluetooth Interferes.

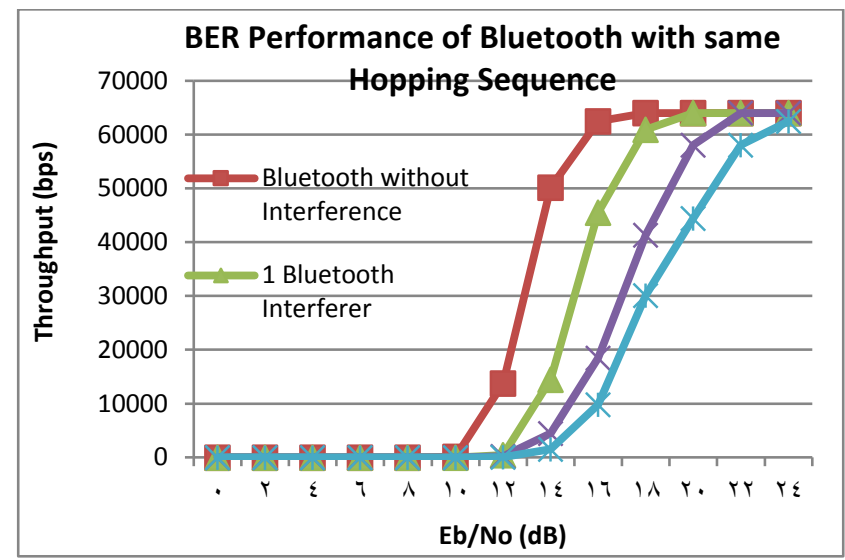

Fig. 4: Throughput Performance of Basic Bluetooth with Bluetooth Interference.



Fig. 5: Throughput Performance of Basic Bluetooth with Bluetooth Interference at different Hopping Frequency.

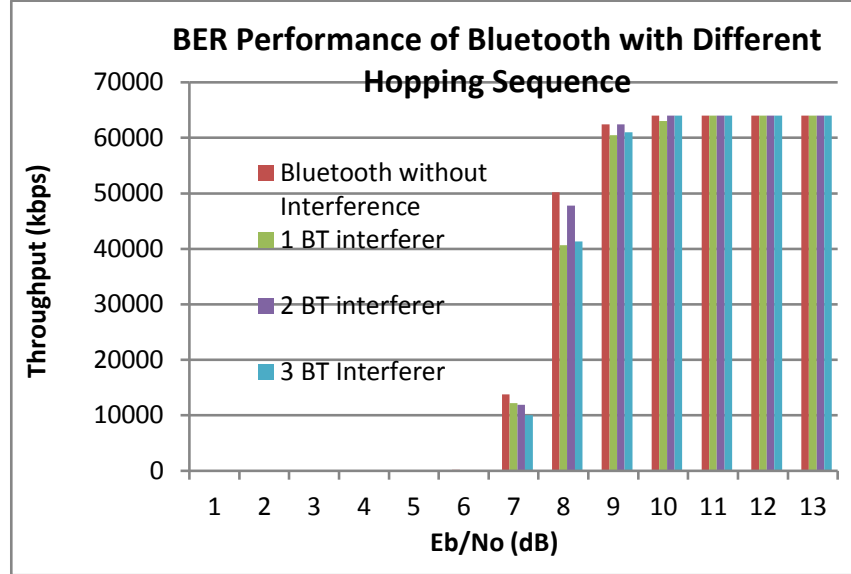

Fig. 6: Throughput Performance of Basic Bluetooth with Bluetooth Interference at different Hopping Frequency.

\section{Conclusion}

In this paper, the performance of Bluetooth investigated inhomogeneous wireless environment. We observed the interference effects of other Bluetooth network that could degrade the performance. From the experimental result obtained shows that BER and throughput get degraded inhomogeneous network. The throughput performance of basic rate Bluetooth in the presence of other Bluetooth with same hopping frequency degraded by $18 \%$, $35 \%$ and $46 \%$ for one, two and three Bluetooth interferes respectively much more than $3.70 \%$ for three Bluetooth interferes at different hopping sequence. It can be concluded that the performance of basic Bluetooth improved by selecting the different hopping frequencies for interfering Bluetooth devices. The BER and throughput performance of basic Bluetooth enhanced by keeping interferer Bluetooth devices beyond 10 meters

\section{Acknowledgement}

Authors are thankful to the Principal, All India Shivaji Memorial's Institute of Information and Technology for providing research facilities. We are also thankful to the experts and friends, who gave the valuable suggestions, directly and indirectly during this work.

\section{References}

[1] Specification of Bluetooth System Core Vol 4.2: [Online] Available: http://www.bluetooth.org.

[2] Jan MIKULKA and Stanislav, "HANUS. Bluetooth and IEEE 802.11 b/g Coexistence Simulation", Radioengineerng, Vol.17. No. 3, (2008), pp. 66-73

[3] S. Zeadally, A. Banda and A. Kumar, "Improving Bluetooth Performance in 802.11 Interference Environments:, Computer Communications, Vol.27, (2004), pp. 616-621.

[4] Jae Sung Roh, "Performance Analysis and Evaluation of Bluetooth Networks in Wireless Channel Environment", International Conference on System and Networks Communication (ICSNC 08), Tahiti, Tahiti, (2006), pp.1-5.

[5] Taiji Kondo, Hiroshi Fujita, Makoto Yoshida and Tamio Saito, "Technology for Wi-Fi/Bluetooth and WiMAX Coexistence", Journal of Science and Technology, Vol.46, No.1, (2010), pp. 7278.

[6] Mathew B. Shoemake, "Wi-Fi (IEEE 802.11b) and Bluetooth Coexistence Issues and Solutions for the $2.4 \mathrm{GHz}$ ISM Band", Texas Instruments, (2001), White Paper: pp.1-17.

[7] Nada Golmie, Nicolas Chevrollier, and Olivier Rebala, "Bluetooth and WLAN Coexistence: Challenges and Solutions", IEEE Wireless Communications, (2003), pp.22-29.

[8] A. S. Shirsat, S. A. Shirsat and D. M. Yadav. Performance of Bluetooth in the Presence of $802.11 \mathrm{~b}$. International Conference on Advances in Communication and Computing (ICACCT). Technically sponsored by IEEE. Sangamner, India. 2018; 117-121. 
[9] Anil Mathew, Nithin Chandrababu, Khaled Elleithy and Syed Rizvi, "IEEE 802.11 and Bluetooth Interference: Simulation and Coexistence", IEEE Computer Society, Seventh Annual Communication Networks and Services Research Conference. Vol.2, No.3, (2010), pp. 140- 149.

[10] R. Challo, A Oladeinde, N. Yilmazer, S. Ozcelik and L. Challo, "An Overview and Assessment of Wireless Technologies and Coexistence of ZigBee, Bluetooth and Wi-Fi Devices", ElsevierProcedia Computer Science. Vol.12, (2012), pp. 386-391.

[11] Chompunut Jantarasorn and Chutima Prommak, "On the Performance Analysis of Coexistence between IEEE 802.11g and IEEE 802.15.4 Networks", World Academy of Science, Engineering and Technology, International Journal of Computer, Information, Systems and Control Engineering. Vol.8, No.7, (2014), pp.1112 1115.

[12] Li Jing, Chen Wanzhong and Zhao Xiaohui, "Achievement of Bluetooth Adaptive Packet Selection Strategies Based on SNR Estimation", Elsevier, the Journal of China Universities of Post and Telecommunications, Vol.1, (2016), pp. 8-13.

[13] A. C. Hsu, D. S. Wei, and C. J. Kuo, "Coexistence Wi-Fi MAC Design for Mitigating Interference Caused by Collocated Bluetooth", IEEE Transactions on Computers, Vol.64, No.2, (2015), pp. 342 352.

[14] C. F. Chiasserini and R. R. Rao, "Coexistence Mechanisms for Interface Mitigation in the 2.4-GHz ISM Band", IEEE Transactions on Wireless Communications. Vol.2, No. 5, (2003), pp.964-975.

[15] P. Desai and B. Ibrahim, "Method and System for Sharing a Single Antenna on Platform with Collocated Bluetooth and IEEE $802.11 \mathrm{~b} / g$ Devices", U. S. Patent No. 9,504,056, November 22, 2016.

[16] M. A. M. Mohamed, A. E. Abu El-Fishway, M. A. R. El Tokhy, F. Shawki and F. E. Abd El-Samie, "Bluetooth Performance Improvement Using Convolutional Codes", Journal of Automation and System Engineering, (2009), pp.1-10.

[17] Seung-Hwan Lee and Yong-Hwan Lee, "Adaptive Frequency Hopping for Bluetooth Robust to WAN Interference", IEEE Communication Letters, Vol.13, (2009), pp.628-631.

[18] M. A. M. El-Bendary, A. E. Abu El-Azm, N. A. El- Fishway, F. Shawki and F. E. Abd El-Samie, "Embedded Throughput Improving of Low-rate EDR Packets for Lower-latency", World Academy of Science, Engineering and Technology Vol.5, (2014), pp.10781087.

[19] M. A. M. El-Bendary and M. A. R. El Tokhy, "Efficient Performance and Lower Complexity of Error Control Schemes for WPAN Bluetooth Networks", Journal of Telecommunication and Information Technology. Vol.4, (2014), pp.100-107.

[20] Weiping Sun, Jonghoe Koo, Seongho Byeon, Woojin Park et al, "BlueCoDE: Bluetooth Coordination in Dense Environment for Better Coexistence", IEEE 25 $5^{\text {th }}$ International Conference on Network Protocols (ICNP), Toronto, Canada, (2017), pp.1 - 10.

[21] B. O. Omijeh and K. Munonye, "Analysis of Bluetooth-Based adhoc Network for Voice Transmission over Local Area Network", International Journal of Scientific \& Engineering Research, Vol.6, No.11, (2015), pp. 460-466.

[22] Muhammad Farrukh Yaqub, Ammar Haider, Iqbal Gondal and Joarder Kamruzzaman, "Self and Static Interference Mitigation Scheme for Coexisting Wireless Networks", Elsevier Computer and Electrical Engineering, Vol.40, (2014), pp. 307 - 318.

[23] M. Gentili, R. Sannino and M. Petracca, "BlueVoice: Voice Communications over Bluetooth Low Energy in the Internet of Things Scenario", Elsevier, Computer Communications. Vol.89, No.90, (2016), pp. 51-59. 